\title{
Microglia Overexpressing the Macrophage Colony- Stimulating Factor Receptor Are Neuroprotective in a Microglial-Hippocampal Organotypic Coculture System
}

\author{
Olivera M. Mitrasinovic, Alicia Grattan, ${ }^{\star}$ Christopher C. Robinson, ${ }^{\star}$ Nicolae B. Lapustea, ${ }^{\star}$ Clara Poon, Heather Ryan, \\ Connie Phong, and Greer M. Murphy Jr \\ Neuroscience Research Laboratories, Department of Psychiatry and Behavioral Sciences, Stanford University School of Medicine, Stanford, California 94305
}

\begin{abstract}
Microglia with increased expression of the macrophage colony-stimulating factor receptor (M-CSFR; $c$-fms) are found surrounding plaques in Alzheimer's disease (AD) and in mouse models for $\mathrm{AD}$ and after ischemic or traumatic brain injury. Increased expression of M-CSFR causes microglia to adopt an activated state that results in proliferation, release of cytokines, and enhanced phagocytosis. To determine whether M-CSFR-induced microglial activation affects neuronal survival, we assembled a coculture system consisting of BV-2 microglia transfected to overexpress the M-CSFR and hippocampal organotypic slices treated with NMDA. Twenty-four hours after assembly of the coculture, microglia overexpressing M-CSFR proliferated at a higher rate than nontransfected control cells and exhibited enhanced migration toward NMDA-injured hippocampal cultures. Surprisingly, coculture with c- $f m s$-transfected microglia resulted in a dramatic reduction in NMDA-induced neurotoxicity. Similar results were observed when cocultures were treated with the teratogen cyclophosphamide. Biolistic overexpression of M-CSFR on microglia endogenous to the organotypic culture also rescued neurons from excitotoxicity. Furthermore, c-fms-transfected microglia increased neuronal expression of macrophage colony-stimulating factor (MCSF), the M-CSFR, and neurotrophin receptors in the NMDA-treated slices, as determined with laser capture microdissection. In the coculture system, direct contact between the exogenous microglia and the slice was necessary for neuroprotection. Finally, blocking expression of the M-CSF ligand by exogenous c-fms-transfected microglia with a hammerhead ribozyme compromised their neuroprotective properties. These results demonstrate a protective role for microglia overexpressing M-CSFR in our coculture system and suggest under certain circumstances, activated microglia can help rather than harm neurons subjected to excitotoxic and teratogen-induced injury.
\end{abstract}

Key words: microglia; hippocampus; organotypic; M-CSF; excitotoxicity; neuroprotection; ribozyme; laser capture microdissection

\section{Introduction}

Microglia play an important role during injury and infection in the nervous system. After ischemic or traumatic injury and in demyelinating diseases, microglia remove cellular debris by phagocytosis (Carson, 2002; Danton and Dietrich, 2003). During CNS bacterial and viral infections, microglia recruit leukocytes, express major histocompatibility complex class II antigens, and present antigen as part of the T-cell immune response (Nelson et al., 2002). Microglia may also inhibit the growth of CNS parasites (Luder et al., 1999). All of these actions are potentially beneficial. However, microglia may have negative effects. There is substantial evidence that activated microglia produce potentially toxic

\footnotetext{
Received 0ct. 6, 2004; revised March 25, 2005; accepted March 25, 2005.

This work was supported by the National Institute of Mental Health (MH 57833) and by the Alzheimer's Association. 0.M.M. is the recipient of an Alzheimer's Association New Investigator Award. We thank Jennifer Rhee, Grace Perez, Mark Rodgers, Angela Chen, and Feifei Zhao for assistance.

*A.G., C.C.R., and N.B.L. contributed equally to this work.

Correspondence should be addressed to Dr. Greer M. Murphy Jr, Associate Professor, Neuroscience Research Laboratories, Department of Psychiatry and Behavioral Sciences, Stanford University School of Medicine, Medical School Lab Surge Building, Room P-104, Stanford, CA 94305-5485. E-mail: gmurphy@leland.stanford.edu. DOI:10.1523/JNEUROSCI.0514-05.2005

Copyright $\odot 2005$ Society for Neuroscience $\quad 0270-6474 / 05 / 254442-\bullet \$ 15.00 / 0$
}

cytokines, reactive oxygen and nitrogen species, prostaglandins, and other factors that may injure neurons already compromised by disease. For example, in Alzheimer's disease (AD), microglia activated by the amyloid $\beta(\mathrm{A} \beta)$ peptide express proinflammatory cytokines, complement, nitric oxide, and superoxide free radicals that contribute to neurodegeneration (McGeer and McGeer, 2002). Similarly, after stroke, activated microglia may accelerate the death of injured neurons by secreting neurotoxic compounds, as well as recruiting large numbers of potentially neurotoxic leukocytes into the brain (Danton and Dietrich, 2003). During CNS infection, microglial-initiated leukocyte invasion, although potentially beneficial at first, may become fulminant and hence play a negative role during CNS infection (Nau and Bruck, 2002). Activated microglia may actually promote the growth of certain CNS neoplasia (Graeber et al., 2002). Clearly, microglia may have both beneficial and detrimental effects in neurologic disease.

Macrophage colony-stimulating factor (M-CSF) is a hematopoietic cytokine that is expressed in brain by neurons and glia. $\mathrm{M}$-CSF induces microglial proliferation and activation and results in expression of a variety of proinflammatory effectors. Interestingly, although M-CSF activates microglia, this cytokine has 
A.

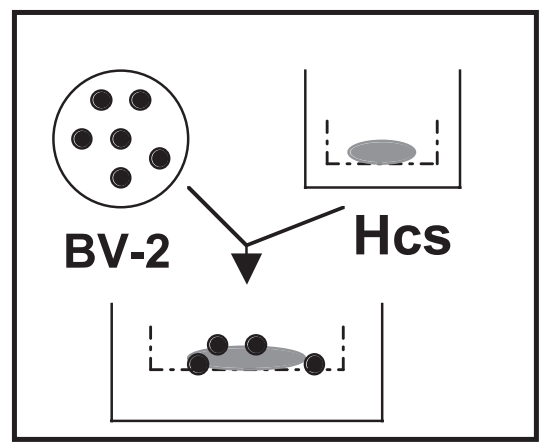

B.
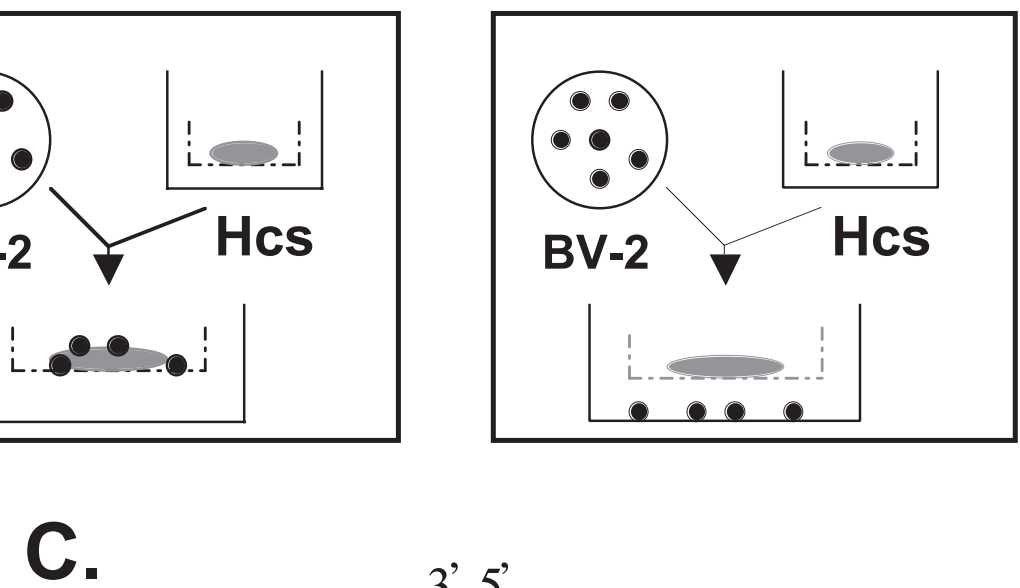

Figure 1. A, Schematic diagram of one-level coculture system. Hcs, Hippocampal cultures. B, Two-level coculture system. C, RNA secondary sequence of the anti-M-CSF hammerhead ribozyme (Rz). Cleavage occurs after nucleotide 266 (M-CSF mRNA sequence NM 007778), as indicated by the arrow.

also been shown to have neuroprotective properties both in vivo and in cell culture (Berezovskaya et al., 1996; Fedoroff et al., 1997; Bruccoleri and Harry, 2000; Vincent et al., 2002a). The M-CSF receptor (M-CSFR) encoded by the proto-oncogene c-fms is upregulated on microglia after traumatic and ischemic brain injury and in $\mathrm{AD}$ and in transgenic mouse models for $\mathrm{AD}$ (Raivich et al., 1998; Wang et al., 1999; Murphy et al., 2000). We showed previously that increased microglial expression of the M-CSFR on cultured microglia and on microglia in organotypic hippocampal cultures induces microglial production of proinflammatory cytokines and nitric oxide, as well as promoting phagocytosis of $\mathrm{A} \beta$ (Mitrasinovic and Murphy, 2002, 2003; Mitrasinovic et al., 2004). In the present study, we sought to determine the effects of microglial overexpression of c-fms on the survival of neurons subjected to excitotoxic injury and to injury induced by the teratogen cyclophosphamide (CP). Increased microglial M-CSFR expression has been linked to CP neurotoxicity (Hao et al., 2002). Because the endotoxin lipopolysaccharide (LPS) has been used to stimulate microglial activation and microglial-induced neurotoxicity (Kim and Ko, 1998; Wang et al., 2002; Xie et al., 2004), we compared the effect of microglia overexpressing the M-CSFR on neuronal survival to that of microglia treated with LPS.

\section{Materials and Methods}

Microglial-hippocampal cocultures. The coculture system consisted of exogenous BV-2 mouse microglial cells and rat organotypic hippocam- pal cultures, as described previously (Mitrasinovic et al., 2001). BV-2 cells maintain many of the phenotypic features of primary microglial cells [for relevant citations, see Mitrasinovic et al. (2004)]. Cells were cultured as described previously (Mitrasinovic et al., 2001) and were used for experiments at a passage number of $<15$. Hippocampal organotypic slice cultures of $400 \mu \mathrm{m}$ in thickness were prepared from 7 -dold neonatal rats and cultured on Millicell-CM membranes (Millipore, Bedford, MA) in sixwell dishes, as described previously (Mitrasinovic et al., 2001). Hippocampal cultures were maintained for 2 weeks before experimentation to reduce microglial activation secondary to the explant procedure. A one-level coculture system was assembled as described previously (Mitrasinovic et al., 2001) by adding BV-2 cells onto the Millicell membrane supporting the hippocampal cultures (Fig. $1 A$ ). The initial seeding density of BV- 2 cells was $4 \times 10^{3}$ cells/ $\mathrm{cm}^{2}$. In the one-level system, BV-2 cells were in direct contact with the organotypic culture. A two-level coculture system contained the same number of exogenous BV-2 microglia, but cells were seeded underneath the insert and adhered to the bottom of the tissue-culture plate (Fig. $1 B)$. In the two-level system, BV-2 cells were not in direct contact with the organotypic culture.

Transfections. Microglial transfections were performed using a Lipofectamine Plus (Invitrogen, San Diego, CA) protocol essentially as described previously (Mitrasinovic et al., 2001). Briefly, BV-2 cells were grown to $65 \%$ confluency $\left(\sim 5 \times 10^{4}\right.$ cells $)$ in six-well culture plates and treated with the plasmid complex containing $0.2 \mu \mathrm{g}$ of simian virus 40 -promoted c-fms (pTK1) plasmid or the pZeoSV control plasmid (lacking c-fms cDNA) preincubated with the Li-

pofectamine Plus reagent. Another control condition was BV-2 cells treated with transfection medium containing Lipofectamine Plus alone. We showed previously that transfection of BV-2 cells with the pTK1 c- $f m s$ plasmid results in an $\sim 75$-fold increase in M-CSFR mRNA, as well as increased M-CSFR protein, and a 2.2-fold increase in microglial expression of the ligand, M-CSF (Mitrasinovic et al., 2001). Transfection efficiency is $\sim 90 \%$ (data not shown). To compare the effects of c-fms transfection with that of endotoxin, a classical stimulus for microglial activation that can induce microglial injury to monotypic neuronal cultures (Kim and Ko, 1998; Wang et al., 2002; Xie et al., 2004), some BV-2 cells were treated with $5 \mu \mathrm{g} / \mathrm{ml}$ LPS (Sigma, St. Louis, MO). After $24 \mathrm{~h}$ of transfection or LPS treatment, cells were detached from the wells by mechanical pipetting, quantified, and used for assembly of the organotypic coculture.

Excitotoxicity. Neuronal toxicity was induced with NMDA (Tocris Cookson, Ballwin, MO), introduced into hippocampal growth medium at final concentrations of 5 or $100 \mu \mathrm{M}$ at the time of the coculture assembly. Cocultures were treated with NMDA for $24 \mathrm{~h}$, except where indicated otherwise in the figures. Pilot studies showed that c-fms-transfected microglia proliferated rapidly in coculture with NMDA-treated slice cultures. To test whether protection against NMDA toxicity conferred by c-fms-transfected BV-2 cells in coculture might be merely a result of increased cell numbers, we assembled cocultures containing the same density of wild-type BV-2 cells as found in c-fms-transfected cocultures after $24 \mathrm{~h}$ of NMDA treatment $\left(6.0 \times 10^{4} \mathrm{cells} / \mathrm{cm}^{2}\right)$. These were compared with cocultures containing the standard starting density of BV-2 cells $\left(4 \times 10^{3}\right.$ cells $\left./ \mathrm{cm}^{2}\right)$.

Teratogen-induced neurotoxicity. It was reported that conditioned me- 
dium from neurons treated with the neurotoxic teratogen compound CP induces microglial expression of the M-CSFR, and it was hypothesized that this promotes microglial neurotoxicity (Hao et al., 2002). To test directly the role of increased microglial M-CSFR in CP-induced neurotoxicity, cyclophosphamide monohydrate (Sigma) was dissolved in nuclease-free water (Promega, Madison, WI) at a concentration of 10 $\mu \mathrm{g} / \mathrm{ml}$ and was added to the coculture at a final concentration of $1 \mu \mathrm{g} / \mathrm{ml}$ for $30 \mathrm{~min}$ at $37^{\circ} \mathrm{C}$ after assembly of the coculture system (Hao et al., 2001, 2002). Seeding density and coculture conditions were the same as described above for NMDA experiments.

Propidium iodide labeling. At the end of neurotoxin treatment, $2.5 \mu \mathrm{l}$ of propidium iodide solution (PI; $1 \mathrm{mg} / \mathrm{ml}$; Molecular Probes, Eugene, $\mathrm{OR}$ ) was added to the growth medium $(1.2 \mathrm{ml})$, and coculture plates were returned to the incubator for an additional $30 \mathrm{~min}$. Labeling reactions were stopped by removing the growth medium, after which sections were washed two times with PBS buffer and then fixed with $4 \%$ paraformaldehyde for $20 \mathrm{~min}$ at room temperature before finally being washed four times with PBS. The Millicell membrane was cut from the insert and mounted on a glass slide using the SlowFade Antifade kit (Molecular Probes). Microscopy was performed on an Axioskop 2FS microscope (Carl Zeiss, Jena, Germany) in the cyanine 3 channel with a $10 \times$ objective. After image acquisition, quantitative analysis was performed with MetaMorph 5.0 software (Universal Imaging Corporation, West Chester, PA). PI fluorescence was quantified as integral regional density, which corresponds to the intensity of the PI emitted fluorescence per unit area after subtraction of the background fluorescence. All comparative measurements were taken using identical acquisition settings and mercury lamp intensity. Mercury lamp intensity was preset based on the highest intensity image to prevent pixel saturation.

FluoroJade staining. After $24 \mathrm{~h}$ of coculture, hippocampal slices with BV-2 cells were fixed in $4 \%$ paraformaldehyde, washed three times with PBS, and cryoprotected at $4^{\circ} \mathrm{C}$ in $20 \%$ sucrose before freezing. Cultures were then sectioned on a cryostat at $15 \mu \mathrm{m}$ and mounted on glass slides. FluoroJade staining was performed following the protocol of Schmued et al. (1997). Slides were first air dried for $30 \mathrm{~min}$, then treated with $100 \%$ ethanol for $3 \mathrm{~min}$, 70\% ethanol for $1 \mathrm{~min}$, and washed with distilled water $\left(\mathrm{dH}_{2} \mathrm{O}\right)$ for $1 \mathrm{~min}$. Subsequently, sections were incubated with $0.06 \%$ potassium permanganate for $15 \mathrm{~min}$ with gentle shaking and then washed with $\mathrm{dH}_{2} \mathrm{O}$ for 1 min. FluoroJade (Chemicon, Temecula, CA) solution was prepared as $0.001 \%$ in $\mathrm{dH}_{2} \mathrm{O}$ containing $0.1 \%$ acetic acid. Slides were incubated with the FluoroJade solution for $30 \mathrm{~min}$ at room temperature and then washed three times with distilled $\mathrm{H}_{2} \mathrm{O}$. After air drying for $30 \mathrm{~min}$, slides were rapidly immersed in xylene and then coverslipped before confocal microscopy.

Biolistic transfections of the organotypic cultures. Hippocampal sections were prepared as described above and after $7 \mathrm{~d}$ were biolistically transfected with a CD11b-c-fms plasmid or a CD11b-enhanced green fluorescent protein (EGFP) construct or the pZeoSV control vector using a Helios Gene Gun system, as described previously (Mitrasinovic et al., 2004). The CD11b promoter specifically targets microglia endogenous to the organotypic hippocampal cultures and results in increased expression of microglial M-CSFR. After biolistic transfection, slices were treated with $100 \mu \mathrm{M}$ NMDA and returned to the incubator. After $24 \mathrm{~h}$, sections were treated with PI for neurotoxicity assessment.

Cell proliferation assay. The number of exogenous microglia in coculture was monitored at 24 and $48 \mathrm{~h}$. BV-2 microglia were removed from the coculture by trypsinization for $4 \mathrm{~min}$ and then collected by centrifugation at 13,000 rpm for $4 \mathrm{~min}$. Cells were resuspended in PBS buffer and quantified with an automatic particle counter (Beckman Coulter, Miami, FL) using $4 \mu \mathrm{m}$ as the particle threshold as described previously (Mitrasinovic et al., 2001). Each sample was counted three times, and each treatment was repeated three times.

$M$-CSF-deficient microglia. An anti-M-CSF hammerhead ribozyme catalytic RNA oligonucleotide was designed with Mfold software (Zuker, 2003). The sequence of the ribozyme is shown in Figure $1 C$ (custom synthesized; Dharmacon Corporation, Boulder, CO). BV-2 microglia were grown to $5 \times 10^{4}$ cells per well in a six-well tissue culture plate and treated with $2 \mu \mathrm{g}$ of the anti-M-CSF RNA oligonucleotide incubated with $15 \mu \mathrm{l}$ of Oligofectamine (Invitrogen) reagent at $37^{\circ} \mathrm{C}$ for $30 \mathrm{~min}$. Cells were simultaneously cotransfected with the c-fms plasmid as described above. Control transfections were performed using an RNA oligonucleotide with the same sequence but synthesized in the reverse $5^{\prime}$ to $3^{\prime}$ orientation. After $24 \mathrm{~h}$, cotransfected BV-2 microglia were used for coculture assembly. Subsequent treatments and outcome measures were identical to those described above.

RNA isolation, reverse transcription, and real-time quantitative PCR. Total RNA was extracted from BV-2 cells using Trizol (Invitrogen). Reverse transcription (RT) was performed with SuperScript reverse transcriptase (Invitrogen). To quantify M-CSF and c-fms mRNA in BV-2 cells, we used a real-time PCR assay with SYBR Green (Applied Biosystems, Foster City, CA), as described previously (Mitrasinovic et al., 2001).

Laser capture microdissection. Pure samples of hippocampal neurons from microglial-organotypic cocultures were obtained as described previously (Vincent et al., 2002b), with some modifications. Cultures were frozen on dry ice, sectioned at $15 \mu \mathrm{m}$ thickness, and mounted on glass slides for membrane-based laser capture. To visualize the neuronal layer, sections were stained with hematoxylin as described. Individual neurons were subsequently captured using a Leica Microsystems (Bannockburn, IL) AS LMD laser microdissection system. An average of 30 neuronal cells was collected from each slice culture, evenly distributed throughout the CA1-CA3 neuronal layer. Total RNA was extracted from captured neurons using the Absolutely RNA Microprep kit (Stratagene, Cedar Creek, TX) and reverse transcribed using Sensiscript (Qiagen, Valencia, CA) as described previously (Vincent et al., 2002b). Real-time PCR with SYBR Green was performed as described previously (Mitrasinovic et al., 2001). All PCR was performed in quadruplicate. To confirm that neuronal cells had been captured, we tested for neurofilament heavy chain (NF-H) expression as described previously (Vincent et al., 2002b). To test for glial contamination, RNA samples were assessed with real-time RT-PCR for expression of the astrocyte markers GFAP and S100 $\beta$ and the microglial marker CD11b. We then tested for neuronal expression of M-CSF, M-CSFR, brain-derived neurotrophic factor (BDNF), and the BDNF receptor variants neurotrophic tyrosine kinase receptor type 2 (NTRK2) full-length and NTRK2 T1 (truncated). Primer sequences used for real-time SYBR Green PCR are presented as supplemental material (available at www.jneurosci.org). To determine expression of the fulllength (M55291) and truncated (M55292) NTRK2 splice variants, sequences were aligned, and nonoverlapping domains were used to design primers for differentiation of isoforms. Neuronal RNA samples captured from cocultures containing control, LPS-treated, and c-fms-transfected BV-2 microglia were compared. Gene expression fold changes between experimental conditions were calculated using the standard curve method [user bulletin 2 (1997); Applied Biosystems]. To adjust for RNA loading, we quantified glyceraldehyde-3-phosphate dehydrogenase expression in each sample.

Statistical analyses. Data were analyzed using one- and two-way ANOVAs. Post hoc comparisons between means were performed using the Scheffé correction to control for type 1 error. All data points represent a minimum of three experimental replicates. For neuroprotection experiments, each data point represents a minimum of six replicates, each replicate consisting of data from three individual slice cultures.

\section{Results}

Microglia overexpressing the M-CSFR were found to be neuroprotective. Figure 2 illustrates the effects of overexpression of the M-CSFR by exogenous microglia on NMDA-induced neurotoxicity in organotypic hippocampal cultures. A two-way ANOVA revealed a significant interaction between the presence or absence of NMDA and microglial treatment/transfection condition $(p<$ $0.0001)$. Cocultures containing microglia overexpressing the M-CSFR and treated with NMDA showed significantly less neurotoxicity (Scheffé-corrected $p<0.05$ ) than hippocampal cultures treated with NMDA, NMDA-treated cultures grown with BV-2 cells transfected with the control plasmid, and NMDAtreated cocultures containing exogenous microglia pretreated with LPS. There were no significant differences in neurotoxicity 


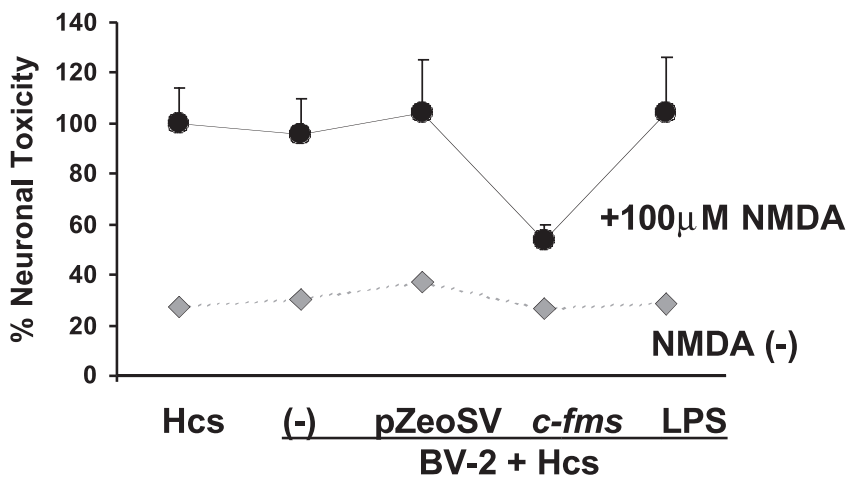

Figure 2. Neuroprotective effects of BV-2 microglia overexpressing M-CSFR in the microglial organotypic coculture system after NMDA treatment. The black data points represent NMDAtreated cocultures, whereas the gray data points represent cocultures not treated with NMDA. The $y$-axis represents PI staining calculated as a percentage of the staining seen hippocampal cultures (Hcs) alone (no microglia) treated with NMDA. This value is compared with a coculture system containing nontransfected microglia treated with transfection medium alone $(-)$, microglia transfected with the control plasmid (pZeoSV), the c-fms plasmid (c-fms), or pretreated with LPS (LPS). Only c-fms-transfected exogenous microglia were neuroprotective. Error bars represent SD.

among the other treatment conditions after NMDA treatment. Among cultures not treated with NMDA, there were no significant differences among treatment conditions.

Examination of PI-stained sections revealed that the decrease in NMDA-induced neurotoxicity occurs within both the CA1 and $\mathrm{CA} 3$ regions of the hippocampus, as well as in the dentate gyrus (Fig. 3A). In PI-stained sections, BV-2 cells surrounding the organotypic culture never showed any signal (Fig. $3 B$; compare with phase-contrast images in Fig. 5A). PI fluorescence was limited to the neuronal layer, and no signal was observed in neuropil regions of the hippocampus despite the presence of numerous overlying BV-2 cells. To verify that PI signal was caused by nerve-cell injury and not caused by PI uptake by other cells endogenous to the organotypic culture, we examined FluoroJade as a highly specific marker for neuronal injury. Results showed strong FluoroJade staining in sections from hippocampal slices treated with NMDA and cultured with wild-type BV-2 cells (Fig. $3 D$ ) but little staining in slices cocultured with BV-2 cells overexpressing the M-CSFR (Fig. 3C).

Because LPS-pretreated BV-2 cells failed to protect neurons from excitotoxic injury in coculture, we examined the expression profile of these cells. Previously, we showed that LPS induces the expression of proinflammatory cytokines such as macrophage inflammatory protein- $1 \alpha$ (MIP- $1 \alpha$ ) by BV-2 cells (Murphy et al., 1995). Transfection with the c-fms construct also induces MIP- $1 \alpha$ production by BV-2 cells (Mitrasinovic et al., 2001). However, in the present study, LPS treatment of BV-2 cells resulted in no significant change in expression of M-CSF $(p>$ 0.10 ) and actually downregulated the expression of M-CSFR by $51 \%(p<0.05)$ in comparison with untreated cells. This is in contrast to c-fms transfection, which results in increased M-CSF and M-CSFR expression (Mitrasinovic et al., 2001).

To test whether neuroprotection conferred by c-fmstransfected microglia occurred only in the coculture system, we used biolistics to induce c-fms overexpression in microglia endogenous to the organotypic culture, as demonstrated previously (Mitrasinovic et al., 2004). Biolistic transfection with the CD11b/ c- $f m s$ construct resulted in a significant average reduction of over $94 \%$ in neurotoxicity compared with control plasmid and CD11b/EGFP transfections (Fig. 4) (Scheffé-corrected $p<0.05$ ).
In fact, there was no significant difference between mean CD11b/ c-fms-induced PI staining and that observed in untreated organotypic cultures.

We observed increased numbers of c-fms transfected BV-2 cells near organotypic slices in the coculture system after NMDA treatment (Fig. 5A, middle). In untreated cocultures, BV-2 cells transfected to overexpress c-fms showed significantly greater proliferation after 24 and $48 \mathrm{~h}$ than both control vector-transfected cells and LPS-treated cells (Fig. 5B, left) (Scheffé-corrected $p<$ $0.05)$. A similar pattern was seen in coculture with organotypic slices treated with NMDA (Fig. 5B, right), although at both 24 and $48 \mathrm{~h}, \mathrm{c}$-fms-induced proliferation was significantly greater than in cocultures not treated with NMDA ( $t$ tests; $p<0.01$ for both).

We tested whether neuroprotection was a function of cell density by coculturing wild-type BV-2 cells at a density equivalent to that observed with c-fms-transfected cells, which proliferate rapidly. NMDA-induced neurotoxicity in cocultures containing a higher density of wild-type BV-2 cells was $84.4 \%$ (SD, 35.0) of that in cocultures with the standard starting density. This difference was not statistically significant. To test whether c-fmsinduced neuroprotection might be a result of nonspecific activation of BV-2 cells in combination with increased cell density, we compared cocultures containing LPS-treated BV-2 cells using the standard starting density to cocultures containing a density typically present in cocultures with c-fms-transfected BV-2 cells. NMDA-induced neurotoxicity in cocultures containing an increased density of LPS-treated BV-2 cells was $93.26 \%$ (SD, 20.5) of that in cocultures containing the standard BV-2 starting density. This difference was not statistically significant.

It has been proposed that increased microglial M-CSF and $\mathrm{M}$-CSFR expression is a cause of CP-induced neurotoxicity (Hao et al., 2001, 2002). We directly tested the role of increased M-CSFR expression on CP-induced neuronal injury. Overexpression of c-fms rendered BV-2 cells neuroprotective in coculture with CP-injured slices. Neuronal injury in cocultures containing c-fms-transfected BV-2 cells was decreased by an average of $98 \%$ in comparison with cocultures containing wild-type or control plasmid-transfected BV-2 cells (Fig. 6A) (Scheffécorrected $p<0.05$ ) but was not significantly different from that observed in untreated slice cultures. We also noted increased proliferation of c-fms-transfected BV-2 cells when cultured with $\mathrm{CP}$-injured slices (data not shown). Rather than promoting CPinduced neurotoxicity, microglial overexpression of the M-CSFR may be protective. These results also show that the neuroprotective effect of microglial M-CSFR overexpression is not limited to NMDA-induced injury.

To determine whether the neuroprotective effects of c-fmstransfected BV-2 cells were dependant on close proximity between exogenous BV-2 microglia and cells endogenous to the slice culture, we compared the direct coculture system (onelevel) (Fig. 1A) to cocultures in which the BV-2 cells were grown on the bottom of the culture plate well and not in contact with the organotypic culture grown on the Millicell membrane above (two-level) (Fig. 1 B). Results showed that c-fms-transfected BV-2 cells in the two-level system provided no neuroprotection in comparison with control or LPS-treated BV-2 cells (Fig. $6 B$, right plot) (ANOVA; $p>0.10$ ). In parallel one-level cocultures with c-fms-transfected BV-2 cells, the characteristic decrease in NMDA neurotoxicity was observed as expected (Fig. $6 B$, left plot) (Scheffé-adjusted $p<0.05$ ).

To characterize the transcriptional response of neurons subjected to excitotoxic injury in coculture with exogenous micro- 
glial cells, we used laser capture microdissection. We found a marked increase in expression of M-CSF and the M-CSFR in neurons captured from NMDA-treated hippocampal cultures grown with microglia overexpressing the M-CSFR compared with cultures grown with control or LPS-treated BV-2 cells (Fig. 7A,B) (Scheffé-adjusted $p<0.05$ ). LPS-treated BV-2 cells resulted in a significant increase in neuronal BDNF (Fig. 7C) (Schefféadjusted $p<0.05$ ), whereas the increase induced by microglial M-CSFR overexpression was not statistically significant. Pretreatment of BV-2 cells with LPS resulted in a significant increase in the neuronal expression of the NTRK2 full-length isoform, but coculture with cells overexpressing the M-CSFR caused a further significant increase in neuronal expression of this isoform (Fig. 7D) (Scheffé-adjusted $p<0.05)$. Significantly increased expression of the BDNF receptor isoform NTRK2 T1 was seen only in cultures containing BV-2 cells overexpressing the M-CSFR (Figs. 7E) (Scheffé-adjusted $p<$ 0.05), whereas the LPS-induced change was not significant. The purity of the captured neuronal cells was verified with realtime RT-PCR. Whereas expression of $\mathrm{NF}-\mathrm{H}$ was strong $\left(\mathrm{C}_{\mathrm{T}}\right.$ value of 28 cycles), real-time RT-PCR signals for the glial markers GFAP, S100 $\beta$, and CD11b were not above background levels.

To test whether M-CSF expression by c-fms-transfected BV-2 microglia in the coculture system was essential for neuroprotection, we used a hammerhead ribozyme to decrease M-CSF production by the exogenous c-fms-transfected cells. Results showed an average $56 \%$ decrease in M-CSF mRNA by BV-2 cells treated with the M-CSF ribozyme, which was significantly less than that for control cells and significantly less than that for cells treated with a control ribozyme (Fig. 8A) (Scheffécorrected $p<0.05$ for both comparisons). In cocultures treated with NMDA, after transfection of BV-2 cells with the M-CSF hammerhead ribozyme, there was an average 53\% decrease in c-fms-induced neuroprotection (Fig. 8 B) (Scheffé-corrected $p<$ $0.05)$. There was no significant loss of neuroprotection in cocultures with c-fms-transfected BV-2 cells treated with the control ribozyme. Because the M-CSF ribozyme inhibited microglial proliferation, we also tested cocultures that contained the same density of ribozyme-treated BV-2 cells as was found in a typical coculture with c-fms-transfected cells. Increased BV-2 cell density did not significantly change the inhibitory effects of the ribozyme on c-fms-induced microglial neuroprotection.

\section{Discussion}

Our results demonstrate that in a microglial-organotypic coculture system and after biolistic transfection of microglia in organotypic cultures, overexpression of the M-CSFR on microglia protects neurons in the slice cultures from excitotoxic and
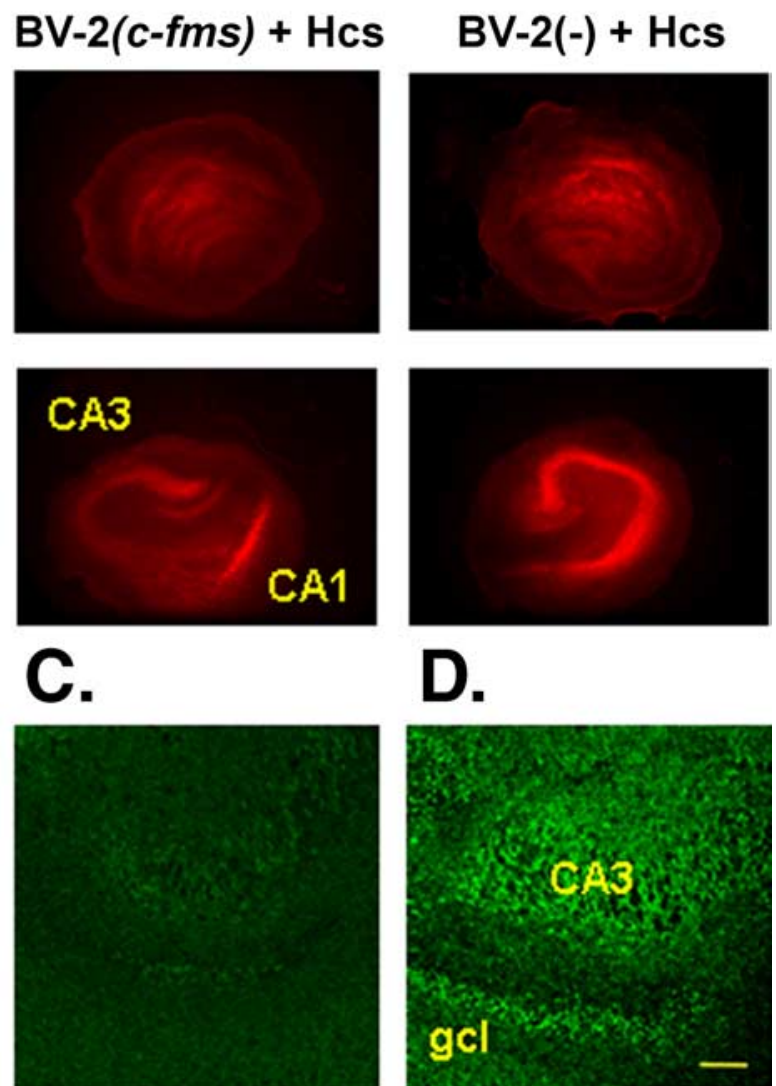

Figure 3. PI labeling of neurotoxicity in the microglial-hippocampal coculture system. $\boldsymbol{A}$, Left column, Hippocampal cultures Hcs) treated with 5 or $100 \mu \mathrm{m}$ NMDA, no BV-2 cells. Middle column, Hippocampal cultures treated with 5 or $100 \mu \mathrm{m}$ NMDA and NMDA and cocultured with BV-2 microglia treated with transfection medium alone $(-)$. B , High-power view of the edge of slice, although containing numerous BV-2 cells, shows no PI signal. C, Confocal image of section from a microglial-organotypic 作 image acquisition to make the CA3 and granule cell layer landmarks faintly visible. D, Confocal images of section from sister coculture stained with FluoroJade. The coculture contained wild-type BV-2 cells transfected to overexpress the M-CSFR and was treated with $100 \mu \mathrm{m}$ NMDA. Note the strong neuronal staining in the CA3 and granule cell layers (gcl). Scale bar, $100 \mu \mathrm{m}$.

teratogen-induced injury. We showed previously that M-CSFR overexpression markedly alters microglial cytokine production, including upregulation of M-CSF, the ligand for the M-CSFR. Inhibition of M-CSF expression in exogenous microglia with a hammerhead ribozyme attenuated the neuroprotective effect of microglial M-CSFR overexpression in the coculture system, indicating that autocrine and/or paracrine effects of this cytokine may be important in neuroprotection. The lack of effect with a control ribozyme makes it unlikely that the ribozyme cotransfection procedure alone was responsible for the loss of neuroprotective properties induced by M-CSFR overexpression. Microglia pretreated with LPS, which were not neuroprotective, lacked increased M-CSF expression.

Although there is substantial evidence that activated microglia can have negative effects in neurologic disease (Spranger and Fontana, 1996; McGeer and McGeer, 1998; Glass and Wesselingh, 2001; Bamberger and Landreth, 2002; Garden, 2002), evidence exists that under certain circumstances, microglia can be neuroprotective. Several groups have found that microglia express the glutamate uptake transporter, which may help micro- 


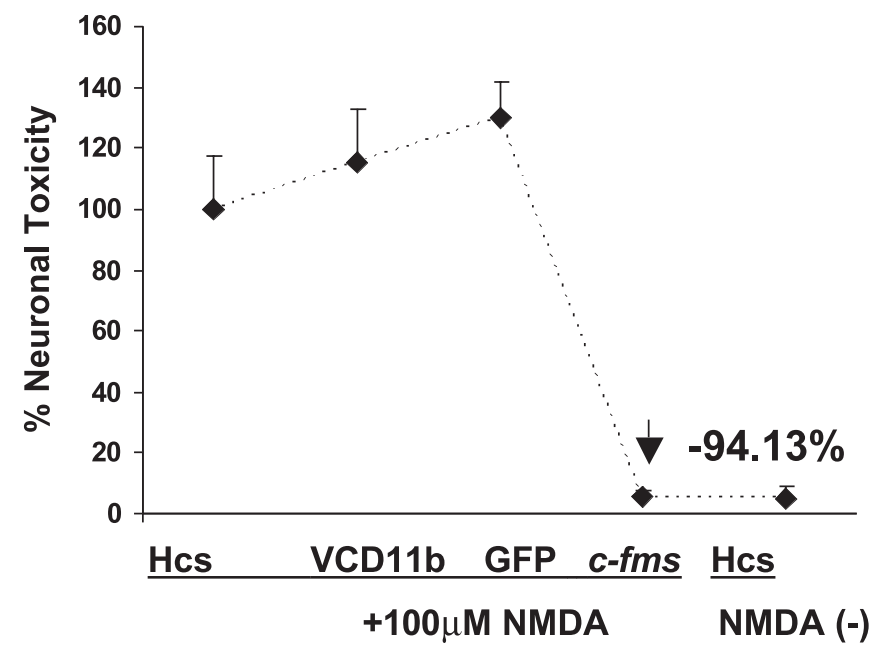

Figure 4. Biolistic expression of the M-CSFR on microglia in organotypic hippocampal cultures protects neurons from NMDA. The $y$-axis shows PI signal expressed as a percentage of nontransfected hippocampal cultures (Hcs). Other conditions included biolistic transfection with the control vector (VCD11b), with the plasmid containing an EGFP CDNA (GFP), with the plasmid resulting in overexpression of M-CSFR on microglia in the slice culture (c-fms), or nontransfected slices not treated with NMDA [Hcs NMDA (-)]. Error bars represent SD.
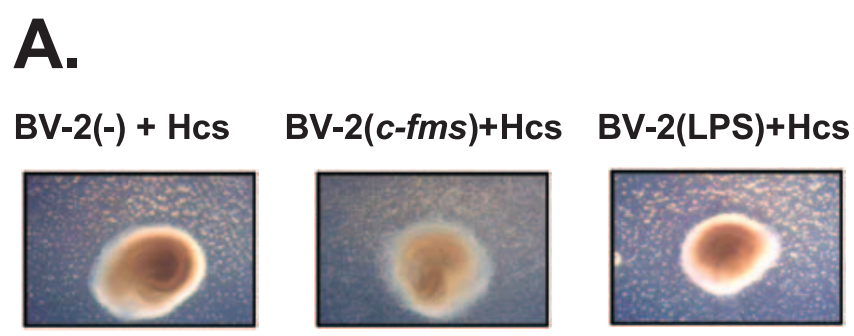

B.

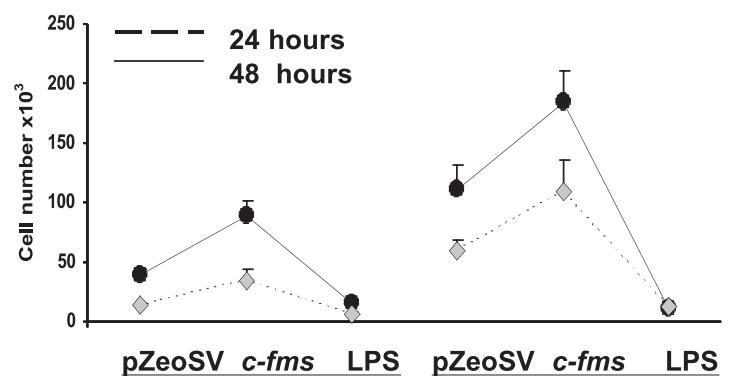

BV-2 + Hcs NMDA (-) BV-2 + Hcs+100 $\mu$ M NMDA

Figure 5. Microglial proliferation induced by c-fms transfection and NMDA treatment in organotypic cocultures. $\boldsymbol{A}$, Phase-contrast images of microglial-organotypic cocultures treated with NMDA. The middle panel shows strong proliferation of BV-2 cells transfected with c-fms $[\mathrm{BV}-2$ (c-fms) + Hcs] compared with nontransfected cells [BV-2 (-) + Hcs] and BV-2 cells pretreated with LPS [BV-2 (LPS) + Hcs]. B, Quantitative analysis of total BV-2 cell numbers per well in cocultures containing BV-2 cells transfected with the control plasmid (pZeoSV), the c-fms plasmid, or pretreated with LPS, with (right) and without (left) the addition of NMDA, after 24 and $48 \mathrm{~h}$. Hcs, Hippocampal cultures. Error bars represent SD.

glia protect nerve cells during excitotoxic injury (Kondo et al., 1995; Lopez-Redondo et al., 2000; Nakajima et al., 2001; Schwartz et al., 2003). Tong et al. (2000) demonstrated that in a coculture model, migration of macrophages toward neurons after administration of the human immunodeficiency virus-related

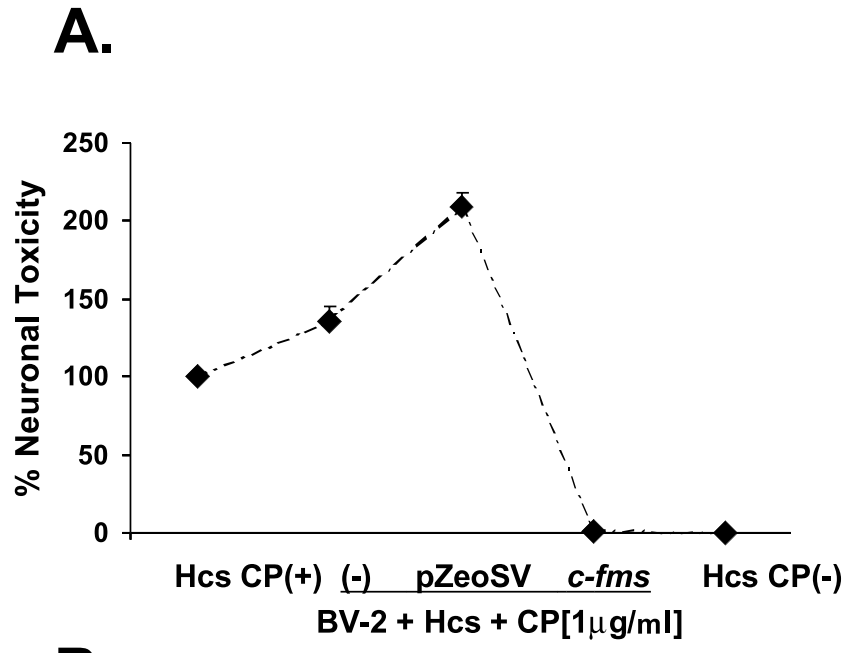

B.

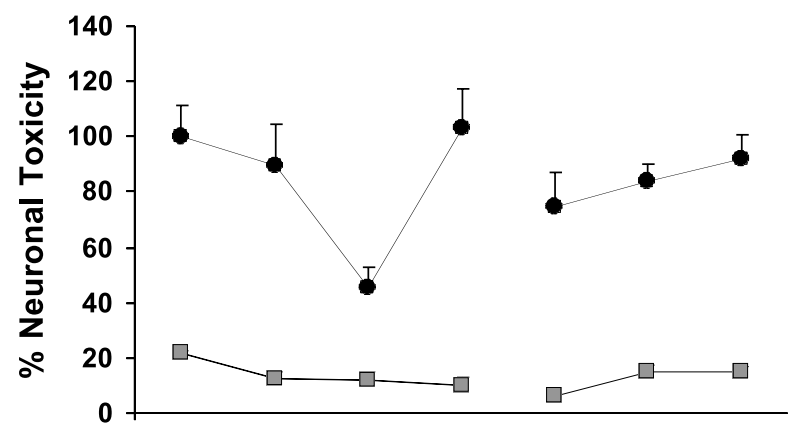

\section{Hcs BV-2(-) c-fms LPS BV-2(-) c-fms LPS Hcs 1-level \\ Hcs 2-level}

Figure 6. A, Neuroprotection by c-fms-transfected exogenous microglia in the microglialorganotypic coculture system after treatment with the teratogen $C P$. Data points represent $\mathrm{PI}$ signal for $(P$-treated hippocampal cultures alone $[\mathrm{Hcs} C P(+)]$ and cocultures with nontransfected BV-2 cells (-), control vector transfected (pZeoSV), and c-fms transfected (c-fms). Also shown are results for untreated hippocampal cocultures containing nontransfected BV-2 cells ( $\mathrm{Hcs}(\mathrm{P}-)$. All data are expressed relative to PI signal in Hcs alone treated with CP. B, Loss of neuroprotection by BV-2 microglia overexpressing the M-CSFR in a two-level coculture system. Black data points represent PI signal in NMDA-treated cultures, whereas gray data points show signal for cocultures not treated with NMDA. All data are expressed as a percentage of PI signal in hippocampal cultures (Hcs) alone treated with NMDA. BV-2 (-), Nontransfected cells; c-fms, c-fms-transfected cells; LPS, LPS-pretreated BV-2 cells. Error bars represent SD.

toxin platelet-activating factor was neuroprotective. Watanabe et al. (2000) found that microglial supernatants protected dissociated neurons from excitotoxic injury. In another coculture model, microglial-neuronal contact changed the microglial phenotype from toxic to protective (Zietlow et al., 1999). Grafting of microglia into injured spinal cord results in neurite outgrowth and other regenerative neuronal responses (Rabchevsky and Streit, 1997). Microglial activation was recently associated with better neuronal survival in an optic nerve crush injury model (Shaked et al., 2004), possibly through recruitment of neuroprotective T-cells to the site of CNS injury (Schwartz and Kipnis, 2004). Non-neuronal cells, including microglia, appear to protect neurons in an animal model for amyotrophic lateral sclerosis (Clement et al., 2003). This and other evidence has led some authors to conclude that an entirely negative role for microglia is probably incorrect (Streit, 2002; Nakajima and Kohsaka, 2004). 
Microglial cytokines may have neurotrophic effects under certain conditions. For example, it was shown recently that microglial tumor necrosis factor- $\alpha$ release induced by activation of the microglia $\mathrm{P}_{2} \mathrm{X}_{7}$ receptor is neuroprotective (Suzuki et al., 2004). We found previously that M-CSF applied directly to organotypic hippocampal cultures protects against excitotoxicity (Vincent et al., 2002a), and similar results have been obtained in vivo (Berezovskaya et al., 1996). Likewise, deletion of M-CSF expression renders neurons vulnerable to injury (Berezovskaya et al., 1995; Bruccoleri and Harry, 2000). The mechanism for M-CSF neuroprotection remains unclear. For microglia transfected to overexpress the M-CSFR cocultured with hippocampal slices, microglial M-CSF could be directly neuroprotective. Or, M-CSF could stimulate exogenous microglia to produce neuroprotectants by autocrine or paracrine mechanisms, or it could stimulate non-neuronal cells in the slice culture to express neuroprotectants or to decrease production of neurotoxins.

Microglial cytokines could also modify gene expression in neurons. Using pure samples of laser-captured slice culture hippocampal neurons, we found that only neuroprotective c-fms-transfected BV-2 cells induced increased expression of neuronal M-CSF and M-CSFR in NMDAtreated cultures. This may result in neuronal self-protection through autocrine and paracrine M-CSF signaling. Some, but not all, studies have reported neuronal expression of the M-CSFR that varies depending on age and anatomic region (Murase and Hayashi, 1998; Raivich et al., 1998). Injury induces neuronal M-CSFR expression locally (Wang et al., 1999). In our coculture system, excitotoxic injury strongly induced neuronal M-CSFR expression when activated microglia are present, which could augment the neurotrophic properties of microglial M-CSF.

Microglia may induce other potentially self-protective neuronal responses. Activation of NMDA receptors increases expression of BDNF and subsequent activation of NTRK2, resulting in neuroprotection in cerebellar granule cell cultures via an autocrine survival loop (Jiang et al., 2003). In our coculture system, only LPS-treated BV-2 cells significantly increased neuronal BDNF expression in coculture. Yet, neurons from cocultures containing c-fms-transfected microglia showed significantly greater expression of the NTRK2 full-length and T1 isoforms than did neurons from cultures containing LPS-pretreated cells. Whereas BDNF binding to full-length NTRK2 containing the tyrosine kinase domain is classically associated with neuroprotection (Rossler et al., 2004), the NTRK2 T1 truncated receptor is said to inhibit the effects of the full-length form (Haapasalo et al., 2002). However, NTRK2 T1 has been shown recently to promote dendritic growth (Yacoubian and Lo, 2000; Hartmann et al., 2004), indicating ligand-indepedent trophic effects of this iso-
B.
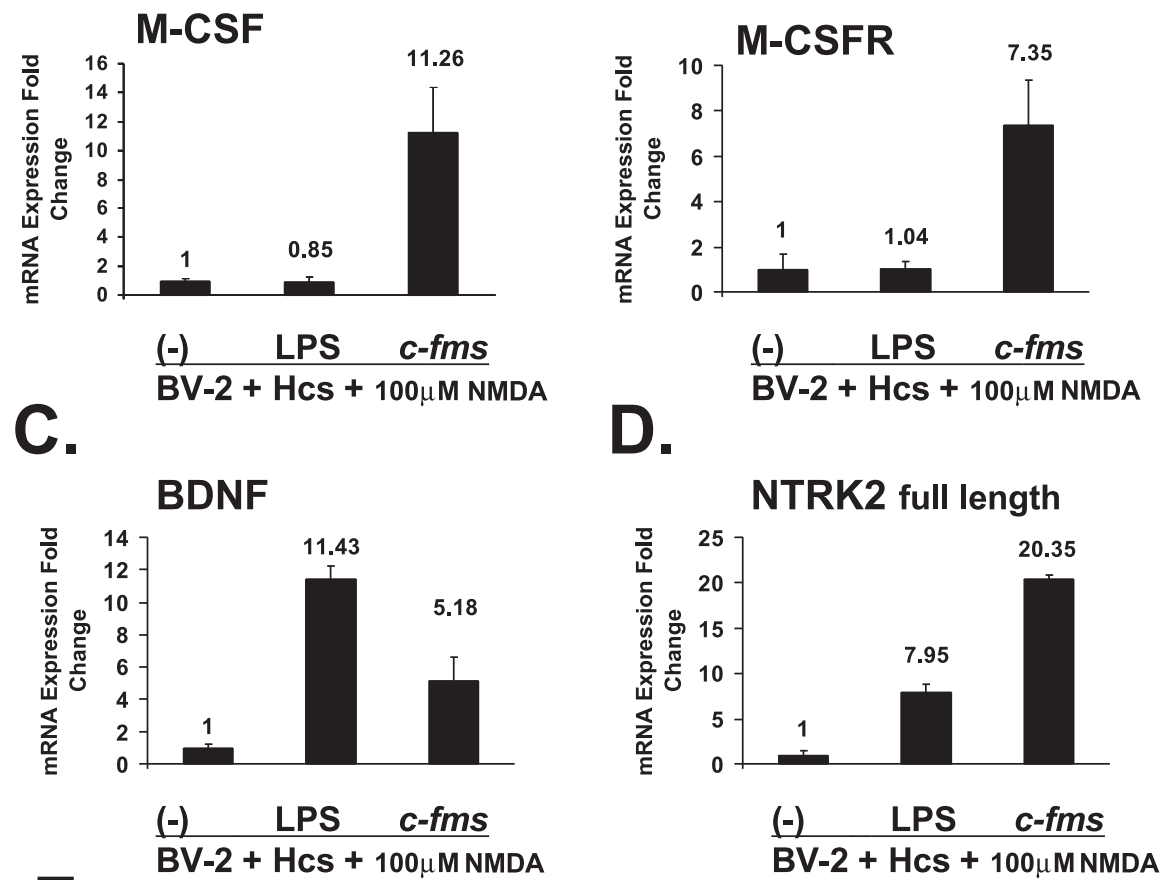

BV-2 + HCs + 100 $\mu$ M NMDA A-E show results from neurons obtained with laser capture microdissection of cocultures. Data are presented for neurona are shown for LPS-pretreated and c-fms-transfected BV-2 cells relative to expression levels in cocultures containing nontransfected BV-2 cells (-). Hcs, Hippocampal cultures. Error bars represent SD.

form. Increased neuronal neurotrophin receptor expression induced by c-fms-transfected microglia could serve to protect nerve cells from excitotoxicity.

Conditioned medium from neurons injured by the teratogen $\mathrm{CP}$ was reported to increase microglial expression of M-CSF and the M-CSFR, leading to production of other inflammatory effectors by microglia (Hao et al., 2001, 2002). It was proposed that this microglial response augments $\mathrm{CP}$-induced neurotoxicity. We directly tested whether microglia with increased expression of $\mathrm{M}-\mathrm{CSF}$ and M-CSFR resulted in neuronal injury. In our system, increased expression of M-CSF and its receptor by microglia actually protected neurons from CP-induced injury. These results demonstrate that microglial activation need not result in neurotoxicity and that neuroprotection induced by microglial overexpression of the M-CSFR occurs with at least two distinct forms of neurotoxicity: NMDA and CP.

We considered the possibility that neuroprotection induced by increased M-CSFR on microglia might occur only in the coculture system. Biolistic transfection was used to overexpress the M-CSFR on microglia endogenous to the slice culture using 
A.
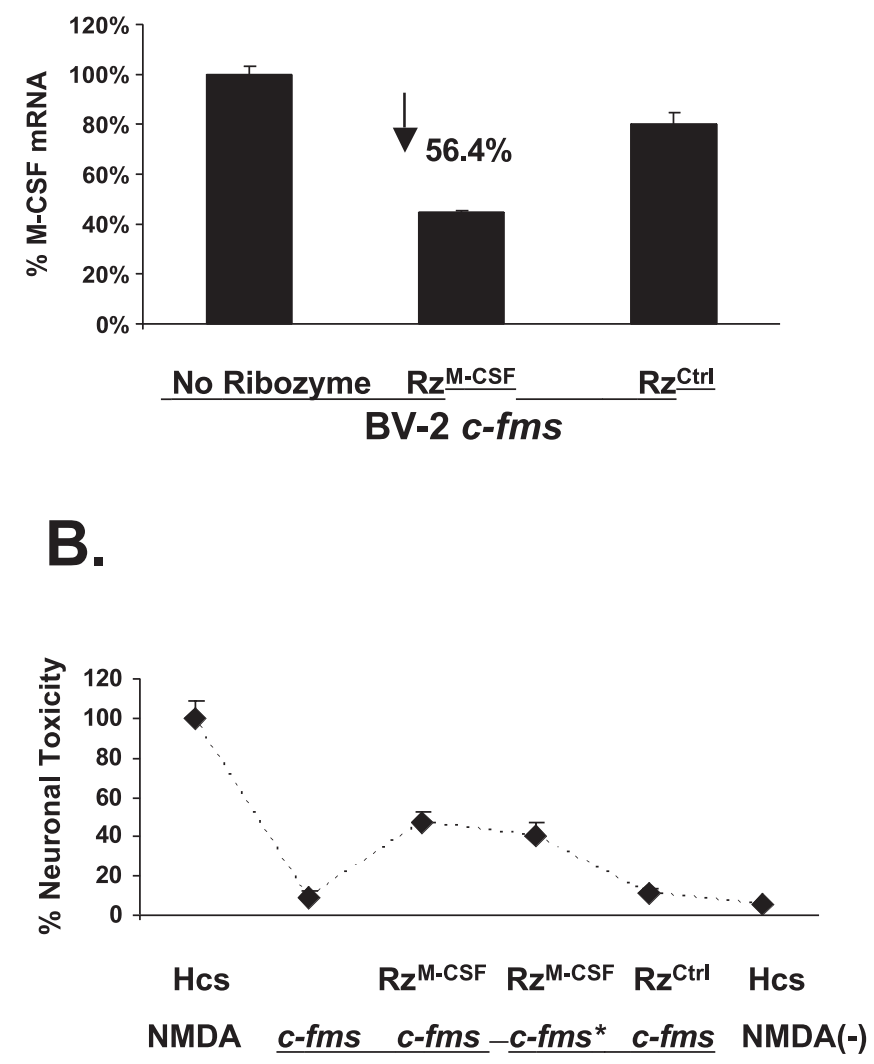

BV-2 + Hcs +100 $\mu$ M NMDA

\section{*Increased density}

Figure 8. $\quad A$, Decreased M-CSF expression by BV-2 microglia in monotypic cultures cotransfected with the $c-f m s$ plasmid and with the anti-M-CSF ribozyme ( $\left.\mathrm{Rz}^{\mathrm{M}-\mathrm{CSF}}\right)$. Data are also presented for a control ribozyme ( $\left.\mathrm{Rz}^{\mathrm{Ctrl}}\right)$. B, Attenuation of neuroprotective effect of BV-2 c-fms transfection in microglial-organotypic cocultures by cotransfection with the anti-M-CSF ribozyme. The $y$-axis represents PI signal relative to that in hippocampal cultures (Hcs) treated with NMDA alone (first data point). Also shown are results for coculture containing c-fmstransfected cells (second data point), c-fms-transfected cells treated with the anti-M-CSF ribozyme (third data point), c-fms-transfected BV-2 cells treated with the anti-M-CSF ribozyme and seeded at increased density (fourth data point), c-fms-transfected cells treated with the control ribozyme (fifth data point), and Hcs without cocultured BV-2 cells and without NMDA (sixth data point). The increased density data point represents cocultures containing numbers of ribozyme-treated cells equal to those typically found in a c-fms-transfected coculture not treated with the ribozyme. Error bars represent SD.

methods that we have described previously (Mitrasinovic et al., 2004). Results were similar to those obtained with the coculture system. Overexpression of EGFP by endogenous microglia did not result in neuroprotection, suggesting that ectopic expression alone is not sufficient to confer neuroprotective properties.

Coculture of microglia overexpressing the M-CSFR with NMDA-treated organotypic cultures resulted in greater proliferation of exogenous microglia than did coculture with untreated slices. A chemotactic response toward the injured slice was also observed. These responses were not observed when exogenous microglia were treated with LPS. We speculated that neuroprotection could be conferred by microglia overexpressing the M-CSFR merely as a result of increased cell numbers. However, when nontransfected BV-2 cells were seeded at an initial density equal to that of c-fms-transfected cells at the end of the experimental period, there was no neuroprotective effect. This suggests that the neuroprotective effects of microglia M-CSFR overexpression are not attributable primarily to increased cell numbers. Interestingly, LPS-treated BV-2 cells, even when seeded at increased density, showed neither a neurotoxic nor a neuroprotective effect in this system.

It is curious that LPS pretreatment of BV-2 microglia did not enhance NMDA neuronal toxicity, because LPS-activated microglia when cocultured with monotypic neurons can result in neurotoxicity (Xie et al., 2004). Aschner et al. (1999) suggested that neurons in dissociated cultures are particularly susceptible to negative effects of microglia as a result of the absence of normal neuronal-glial interactions. In fact, several reports suggest that astrocytic and neuronal factors are key in determining the toxic potential of microglia (Zietlow et al., 1999; Hailer et al., 2001; Smits et al., 2001).

We found that the neuroprotective effect of exogenous microglia overexpressing the M-CSFR occurred only when the microglia were in direct physical contact with the organotypic culture. In the one-level coculture system, some microglia come into direct contact with neurons (Mitrasinovic et al., 2001), although the majority remain on the surface of the slice culture. The local concentration of soluble factors such as M-CSF may be much higher when BV-2 cells are in direct contact with the slice culture than when diluted in the large volume of culture medium of the two-level system. Within tissue, cytokines can likely influence cells within a domain of up to $250 \mu \mathrm{m}$ surrounding the effector cell (Francis and Palsson, 1997), which is approximately the thickness of a mature slice culture. Microglia on the surface of the slice are likely to have paracrine effects on cells located internally. Furthermore, in addition to a soluble form, M-CSF exists as a membrane-bound glycoprotein and extracellular matrix-bound proteoglycan that are biologically active (Fixe and Praloran, 1997). These M-CSF forms participate in cell adhesion and activation (Uemura et al., 1993; Yao et al., 2003) and could be important in neuroprotection. Membrane- or extracellular-bound M-CSF would not affect neurons or other cells in the slice culture in the two-level system, resulting in loss of neuroprotection.

In summary, we demonstrated that microglia overexpressing the M-CSFR protect neurons against excitotoxicity and teratogen-induced injury in a microglial-organotypic coculture system and after biolistic expression of the M-CSFR on microglia endogenous to the slice culture. It is unclear whether microglia showing increased expression of the M-CSFR could be neuroprotective in intact brain. However, because the M-CSFR expression is increased on microglia in $\mathrm{AD}$, additional investigation of the effects of signaling through this receptor on microglial phenotype is warranted.

\section{References}

Aschner M, Allen JW, Kimelberg HK, LoPachin RM, Streit WJ (1999) Glial cells in neurotoxicity development. Annu Rev Pharmacol Toxicol 39:151-173.

Bamberger ME, Landreth GE (2002) Inflammation, apoptosis, and Alzheimer's disease. Neuroscientist 8:276-283.

Berezovskaya O, Maysinger D, Fedoroff S (1995) The hematopoietic cytokine, colony-stimulating factor 1 , is also a growth factor in the CNS: congenital absence of CSF-1 in mice results in abnormal microglial response and increased neuron vulnerability to injury. Int J Dev Neurosci 13:285-299.

Berezovskaya O, Maysinger D, Fedoroff S (1996) Colony stimulating factor-1 potentiates neuronal survival in cerebral cortex ischemic lesion. Acta Neuropathol (Berl) 92:479-486.

Bruccoleri A, Harry GJ (2000) Chemical-induced hippocampal neurode- 
generation and elevations in TNFalpha, TNFbeta, IL-1alpha, IP-10, and MCP-1 mRNA in osteopetrotic (op/op) mice. J Neurosci Res 62:146-155.

Carson MJ (2002) Microglia as liaisons between the immune and central nervous systems: functional implications for multiple sclerosis. Glia 40:218-231.

Clement AM, Nguyen MD, Roberts EA, Garcia ML, Boillee S, Rule M, McMahon AP, Doucette W, Siwek D, Ferrante RJ, Brown Jr RH, Julien J-P, Goldstein LSB, Cleveland DW (2003) Wild-type nonneuronal cells extend survival of SOD1 mutant motor neurons in ALS mice. Science 302:113-117.

Danton GH, Dietrich WD (2003) Inflammatory mechanisms after ischemia and stroke. J Neuropathol Exp Neurol 62:127-136.

Fedoroff S, Berezovskaya O, Maysinger D (1997) Role of colony stimulating factor-1 in brain damage caused by ischemia. Neurosci Biobehav Rev 21:187-191.

Fixe P, Praloran V (1997) Macrophage colony-stimulating-factor (M-CSF or CSF-1) and its receptor: structure-function relationships. Eur Cytokine Netw 8:125-136.

Francis K, Palsson BO (1997) Effective intercellular communication distances are determined by the relative time constants for cyto/chemokine secretion and diffusion. Proc Natl Acad Sci USA 94:12258-12262.

Garden GA (2002) Microglia in human immunodeficiency virus-associated neurodegeneration. Glia 40:240-251.

Glass JD, Wesselingh SL (2001) Microglia in HIV-associated neurological diseases. Microsc Res Tech 54:95-105.

Graeber MB, Scheithauer BW, Kreutzberg GW (2002) Microglia in brain tumors. Glia 40:252-259.

Haapasalo A, Sipola I, Larsson K, Akerman KE, Stoilov P, Stamm S, Wong G, Castren E (2002) Regulation of TRKB surface expression by brainderived neurotrophic factor and truncated TRKB isoforms. J Biol Chem 277:43160-43167.

Hailer NP, Wirjatijasa F, Roser N, Hischebeth GT, Korf HW, Dehghani F (2001) Astrocytic factors protect neuronal integrity and reduce microglial activation in an in vitro model of $\mathrm{N}$-methyl-D-aspartate-induced excitotoxic injury in organotypic hippocampal slice cultures. Eur J Neurosci 14:315-326.

Hao AJ, Dheen ST, Ling EA (2001) Response of amoeboid microglia/brain macrophages in fetal rat brain exposed to a teratogen. J Neurosci Res 64:79-93.

Hao AJ, Dheen ST, Ling EA (2002) Expression of macrophage colonystimulating factor and its receptor in microglia activation is linked to teratogen-induced neuronal damage. Neuroscience 112:889-900.

Hartmann M, Brigadski T, Erdmann KS, Holtmann B, Sendtner M, Narz F, Lessmann V (2004) Truncated TrkB receptor-induced outgrowth of dendritic filopodia involves the p75 neurotrophin receptor. J Cell Sci 117:5803-5814.

Jiang X, Zhu D, Okagaki P, Lipsky R, Wu X, Banaudha K, Mearow K, Strauss KI, Marini AM (2003) N-Methyl-D-aspartate and TrkB receptor activation in cerebellar granule cells: an in vitro model of preconditioning to stimulate intrinsic survival pathways in neurons. Ann NY Acad Sci 993: 134-145 [discussion 993:159-160].

Kim WK, Ko KH (1998) Potentiation of N-methyl-D-aspartate-mediated neurotoxicity by immunostimulated murine microglia. J Neurosci Res $54: 17-26$.

Kondo K, Hashimoto H, Kitanaka J, Sawada M, Suzumura A, Marunouchi T, Baba A (1995) Expression of glutamate transporters in cultured glial cells. Neurosci Lett 188:140-142.

Lopez-Redondo F, Nakajima K, Honda S, Kohsaka S (2000) Glutamate transporter GLT-1 is highly expressed in activated microglia following facial nerve axotomy. Brain Res Mol Brain Res 76:429-435.

Luder CG, Giraldo-Velasquez M, Sendtner M, Gross U (1999) Toxoplasma gondii in primary rat CNS cells: differential contribution of neurons, astrocytes, and microglial cells for the intracerebral development and stage differentiation. Exp Parasitol 93:23-32.

McGeer PL, McGeer EG (1998) Mechanisms of cell death in Alzheimer disease-immunopathology. J Neural Transm Suppl 54:159-166.

McGeer PL, McGeer EG (2002) Local neuroinflammation and the progression of Alzheimer's disease. J Neurovirol 8:529-538.

Mitrasinovic OM, Murphy Jr GM (2002) Accelerated phagocytosis of amyloid-beta by mouse and human microglia overexpressing the M-CSF receptor. J Biol Chem 277:29889-29896.

Mitrasinovic OM, Murphy Jr GM (2003) Microglial overexpression of the
M-CSF receptor augments phagocytosis of opsonized Abeta. Neurobiol Aging 24:807-815.

Mitrasinovic OM, Perez GV, Zhao F, Lee YL, Poon C, Murphy Jr GM (2001) Overexpression of macrophage colony-stimulating factor receptor on microglial cells induces an inflammatory response. J Biol Chem 276:30142-30149.

Mitrasinovic OM, Robinson CC, Tenen DG, Lee YL, Poon C, Murphy Jr GM (2004) Biolistic expression of the macrophage colony stimulating factor receptor in organotypic cultures induces an inflammatory response. J Neurosci Res 77:420-429.

Murase S, Hayashi Y (1998) Expression pattern and neurotrophic role of the c-fms proto-oncogene M-CSF receptor in rodent Purkinje cells. J Neurosci 18:10481-10492.

Murphy Jr GM, Jia XC, Song Y, Ong E, Shrivastava R, Bocchini V, Lee YL, Eng LF (1995) Macrophage inflammatory protein 1-alpha mRNA expression in an immortalized microglial cell line and cortical astrocyte cultures. J Neurosci Res 40:755-763.

Murphy Jr GM, Zhao F, Yang L, Cordell B (2000) Expression of macrophage colony-stimulating factor receptor is increased in the AbetaPP(V717F) transgenic mouse model of Alzheimer's disease. Am J Pathol 157:895-904.

Nakajima K, Kohsaka S (2004) Microglia: neuroprotective and neurotrophic cells in the central nervous system. Curr Drug Targets Cardiovasc Haematol Disord 4:65-84.

Nakajima K, Tohyama Y, Kohsaka S, Kurihara T (2001) Ability of rat microglia to uptake extracellular glutamate. Neurosci Lett 307:171-174.

Nau R, Bruck W (2002) Neuronal injury in bacterial meningitis: mechanisms and implications for therapy. Trends Neurosci 25:38-45.

Nelson PT, Soma LA, Lavi E (2002) Microglia in diseases of the central nervous system. Ann Med 34:491-500.

Rabchevsky AG, Streit WJ (1997) Grafting of cultured microglial cells into the lesioned spinal cord of adult rats enhances neurite outgrowth. J Neurosci Res 47:34-48.

Raivich G, Haas S, Werner A, Klein MA, Kloss C, Kreutzberg GW (1998) Regulation of MCSF receptors on microglia in the normal and injured mouse central nervous system: a quantitative immunofluorescence study using confocal laser microscopy. J Comp Neurol 395:342-358.

Rossler OG, Giehl KM, Thiel G (2004) Neuroprotection of immortalized hippocampal neurones by brain-derived neurotrophic factor and Raf-1 protein kinase: role of extracellular signal-regulated protein kinase and phosphatidylinositol 3-kinase. J Neurochem 88:1240-1252.

Schmued LC, Albertson C, Slikker Jr W (1997) Fluoro-Jade: a novel fluorochrome for the sensitive and reliable histochemical localization of neuronal degeneration. Brain Res 751:37-46.

Schwartz M, Kipnis J (2004) A common vaccine for fighting neurodegenerative disorders: recharging immunity for homeostasis. Trends Pharmacol Sci 25:407-412.

Schwartz M, Shaked I, Fisher J, Mizrahi T, Schori H (2003) Protective autoimmunity against the enemy within: fighting glutamate toxicity. Trends Neurosci 26:297-302.

Shaked I, Porat Z, Gersner R, Kipnis J, Schwartz M (2004) Early activation of microglia as antigen-presenting cells correlates with T cell-mediated protection and repair of the injured central nervous system. J Neuroimmunol 146:84-93.

Smits HA, van Beelen AJ, de Vos NM, Rijsmus A, van der Bruggen T, Verhoef J, van Muiswinkel FL, Nottet HS (2001) Activation of human macrophages by amyloid-beta is attenuated by astrocytes. J Immunol 166:6869-6876.

Spranger M, Fontana A (1996) Activation of microglia: a dangerous interlude in the brain. Neuroscientist 2:293-299.

Streit WJ (2002) Microglia as neuroprotective, immunocompetent cells of the CNS. Glia 40:133-139.

Suzuki T, Hide I, Ido K, Kohsaka S, Inoue K, Nakata Y (2004) Production and release of neuroprotective tumor necrosis factor by $\mathrm{P} 2 \mathrm{X} 7$ receptoractivated microglia. J Neurosci 24:1-7.

Tong N, Perry SW, Zhang Q, James HJ, Guo H, Brooks A, Bal H, Kinnear SA, Fine S, Epstein LG, Dairaghi D, Schall TJ, Gendelman HE, Dewhurst S, Sharer LR, Gelbard HA (2000) Neuronal fractalkine expression in HIV-1 encephalitis: roles for macrophage recruitment and neuroprotection in the central nervous system. J Immunol 164:1333-1339.

Uemura N, Ozawa K, Takahashi K, Tojo A, Tani K, Harigaya K, Suzu S, Motoyoshi K, Matsuda H, Yagita H, Okumura K, Asano S (1993) Bind- 
ing of membrane-anchored macrophage colony-stimulating factor (MCSF) to its receptor mediates specific adhesion between stromal cells and M-CSF receptor-bearing hematopoietic cells. Blood 82:2634-2640.

Vincent VA, Robinson CC, Simsek D, Murphy GM (2002a) Macrophage colony stimulating factor prevents NMDA-induced neuronal death in hippocampal organotypic cultures. J Neurochem 82:1388-1397.

Vincent VA, DeVoss JJ, Ryan HS, Murphy Jr GM (2002b) Analysis of neuronal gene expression with laser capture microdissection. J Neurosci Res 69:578-586.

Wang MJ, Lin WW, Chen HL, Chang YH, Ou HC, Kuo JS, Hong JS, Jeng KC (2002) Silymarin protects dopaminergic neurons against lipopolysaccharide-induced neurotoxicity by inhibiting microglia activation. Eur J Neurosci 16:2103-2112.

Wang Y, Berezovska O, Fedoroff S (1999) Expression of colony stimulating factor-1 receptor (CSF-1R) by CNS neurons in mice. J Neurosci Res $57: 616-632$.
Watanabe H, Abe H, Takeuchi S, Tanaka R (2000) Protective effect of microglial conditioning medium on neuronal damage induced by glutamate. Neurosci Lett 289:53-56.

Xie Z, Smith CJ, Van Eldik LJ (2004) Activated glia induce neuron death via MAP kinase signaling pathways involving JNK and p38. Glia 45:170-179.

Yacoubian TA, Lo DC (2000) Truncated and full-length TrkB receptors regulate distinct modes of dendritic growth. Nat Neurosci 3:342-349.

Yao GQ, Wu JJ, Sun BH, Troiano N, Mitnick MA, Insogna K (2003) The cell surface form of colony-stimulating factor-1 is biologically active in bone in vivo. Endocrinology 144:3677-3682.

Zietlow R, Dunnett SB, Fawcett JW (1999) The effect of microglia on embryonic dopaminergic neuronal survival in vitro: diffusible signals from neurons and glia change microglia from neurotoxic to neuroprotective. Eur J Neurosci 11:1657-1667.

Zuker M (2003) Mfold web server for nucleic acid folding and hybridization prediction. Nucleic Acids Res 31:3406-3415. 